\title{
Utility of Immunohistochemistry on Bone Marrow Trephine Biopsy for the Diagnosis and Classification of Acute Leukemia
}

\begin{abstract}
Background: Bone marrow examination is an essential diagnostic tool in acute leukemia for the determination of lineage of blasts by flow cytometry or immunophenotyping. In cases of dry tap or where facilities of flow cytometry is not available, immunohistochemistry (IHC) on trephine biopsy sections can be used as a supportive tool to diagnose and classify acute leukemia. Materials and Methods: The giemsa-stained peripheral smears and bone marrow aspirate/ imprints smears were retrospectively evaluated. IHC was done on trephine biopsy sections using a panel of antibodies which included CD34, MPO, CD117, Tdt, CD3, CD20, PAX5, and CD10 Results: Of the 29 cases diagnosed by IHC on trephine sections, 25 cases could be accurately classified based on the expression of myeloid and lymphoid markers. Majority of the acute lymphoblastic leukemia (ALL) were of B-cell lineage (81.25\%), and almost half of the B-ALL were common acute lymphoblastic leukemia-associated antigen positive. Conclusion: IHC on trephine biopsy facilitates the confirmation and subtyping of leukemia in more than $85 \%$ cases. A limited panel of antibodies using CD34, MPO, TdT, CD3 CD20, PAX 5, and CD10 can be used for subtyping of leukemias in trephine biopsy sections, especially in centers with limited resources. It obviates the need for a repeat bone marrow procedure where material is not available for flow cytometry.
\end{abstract}

Keywords: Acute leukemia, immunohistochemistry, trephine biopsy

\section{Introduction}

The recent World Health Organization classification of acute leukemia is based on the incorporation of morphology, cytochemistry, immunophenotyping, cytogenetics, and molecular genetics to categorize into distinct subtypes. ${ }^{[1]}$ Bone marrow examination is an integral part of the diagnostic work up and provides material for immunophenotyping by flow cytometry or immunohistochemistry (IHC). Flow cytometry is preferred over IHC due to the better preservation of antigens. However, the introduction of new antibodies have overcome these limitations, and now IHC can be used as an alternative method to primarily diagnose and classify acute leukemia, especially in dry tap. ${ }^{[2]}$ In this article, we have attempted to assess the efficacy of IHC on trephine biopsy and select a panel of immunomarkers that will aid in the diagnosis and classification of acute leukemias.

\footnotetext{
This is an open access journal, and articles are distributed under the terms of the Creative Commons Attribution-NonCommercial-ShareAlike 4.0 License, which allows others to remix, tweak, and build upon the work non-commercially, as long as appropriate credit is given and the new creations are licensed under the identical terms.
}

For reprints contact: WKHLRPMedknow_reprints@wolterskluwer.com

\section{Materials and Methods}

All cases of acute leukemia diagnosed on trephine biopsy over a period of 2 years from January 2017 to December 2018 were analyzed retrospectively. The relevant clinical features and complete blood counts were retrieved from the medical records. The giemsa-stained peripheral smears (PSs) were reviewed along with bone marrow aspirate (BMA)/ imprint smears. Cytochemical stains, including Sudan black B (SBB) and periodic acid-Schiff (PAS), were assessed where ever available. Initial diagnosis was made on morphology based on the examination of PS and BMA smears. Imprint smears were examined where ever aspirate was aparticulate. The cases were further sub-typed on cytochemistry where ever possible. Confirmation and subtyping were done on trephine biopsy sections using IHC. Routine hematoxylin- and eosin-stained trephine biopsy sections along with IHC were evaluated in all cases.

How to cite this article: Rani HS, Hui M, Uppin MS, Uppin SG, Sadashivudu G, Paul TR. Utility of immunohistochemistry on bone marrow trephine biopsy for the diagnosis and classification of acute leukemia. Indian J Med Paediatr Oncol 2020;41:683-87.

\section{H Sudha Rani', Monalisa Hui ${ }^{1}$, Megha S Uppin', Shantveer G Uppin ${ }^{1}$, G Sadashivudu², Tara Roshni Paul ${ }^{1}$}

${ }^{1}$ Department of Pathology, Nizam's Institute of Medical Sciences, Hyderabad,

Telangana, India, ${ }^{2}$ Department of Medical Oncology, Nizam's Institute of Medical Sciences, Hyderabad, Telangana, India

Submitted: $23-O c t-2019$

Revised: 25-Mar-2020

Accepted: 01-Jun-2020

Published: 29-Oct-2020

Address for correspondence: Dr. Tara Roshni Paul, Department of Pathology, Nizam's Institute of Medical Sciences, Punjagutta, Hyderabad - 500 082, Telangana, India.

E-mail: troshnip@yahoo.co.in

Access this article online

Website: www.ijmpo.org

DOI: 10.4103/ijmpo.ijmpo_216_19 Quick Response Code:

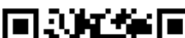




\section{Inclusion criteria}

Cases diagnosed as acute leukemia on trephine biopsy where peripheral blood or BMAs were not available for flow cytometry or yielded inconsistent results were analyzed. These included (i) aparticulate aspirate/dry tap with no blast prominence on PS, (ii) clinically unsuspected cases of leukemia with no blast prominence on PS, (iii) low WBC counts with blasts on PS where blasts could not be gated, and (iv) inconclusive flow cytometry results.

\section{Exclusion criteria}

Cases diagnosed on peripheral blood or BMA where immunophenotyping was done by flow cytometry were excluded. Bone marrow aspiration done in the treated cases of acute leukemia in the postinduction phase to assess the response to therapy was also excluded.

IHC was done using polymer horse radish peroxidase technique on fully automated immunostainer (Xmatrx Elite; Biogenex). The panel of markers included CD 34, MPO, CD117, Tdt, CD3, CD20, PAX5, and CD10 and is listed in Table 1.

\section{Results}

During the study period, a total number of 443 cases of acute leukemia were diagnosed, of which 29 (6.54\%) cases were diagnosed and classified on trephine biopsy. The age of the patients ranged from 6 to 87 years, with a mean of 32.7 years. The patients most commonly presented with fever $(68.9 \%)$, followed by generalized weakness $(29 \%)$, shortness of breath (24\%), jaundice (13\%), and bleeding manifestations $(10 \%)$. The other rare presentation included anorexia, jaw swelling, loss of weight, and cough. Majority of the patients had pallor accounting for $86 \%$. There were five patients who had splenomegaly followed by lymphadenopathy (4 patients) and hepatomegaly (2 patients).

Although PS revealed blasts in four patients, immunophenotyping could not be done as the WBC count was low and the percentage of blasts were very few and could not be gated on immunophenotyping. Another case showed inconsistent result on immunophenotyping, so IHC was done on trephine biopsy for a definite diagnosis. The

\begin{tabular}{lll}
\hline \multicolumn{2}{c}{ Table 1: The panel of immunohistochemical markers } \\
\hline IHC marker & Clone & Suppliers \\
\hline CD34 & QBend/10 & Biogenex \\
MPO & AR496-5R & Biogenex \\
CD117 & T595 & Biogenex \\
TdT & EP266 & Biogenex \\
CD3 & PS1 & Biogenex \\
CD20 & L26 & Biogenex \\
PAX5 & ZP007 & Biogenex \\
CD10 & $56 C 6$ & Biogenex \\
\hline IHC - Immunohistochemistry
\end{tabular}

BMA was scant particulate in seven patients. However, additional material for flow cytometry was not collected in these cases as there was no clinical suspicion of leukemia. Of these, three patients were suspected to have aplastic anemia and one patient each had megaloblastic anemia, tuberculosis, pyrexia of unknown origin, and idiopathic thrombocytopenic purpura.

The BMA was aparticulate in 22 cases. On imprint smears, a definite diagnosis of acute leukemia was made in 14 cases on morphology. Of these 14 cases, cytochemical stains were done in nine cases. There were four cases which showed block positivity for PAS stain and were thus classified as acute lymphoblastic leukemia (ALL). There were six cases which were suspicious of acute leukemia on morphology. Cytochemical stains done in two of the cases which were suspicious of leukemia were noncontributory. In addition, there were two cases which were hypocellular on imprint smears, and one of them showed scattered atypical cells which could not be further categorized.

There were seven cases where scant particles were obtained during aspiration. The aspirate and imprint smears were evaluated simultaneously. On morphology, two cases were diagnosed with acute leukemia, three cases were suspicious of acute leukemia, and two cases were unclassified. Cytochemical stains done in six cases were noncontributory.

The four cases which were hypocellular on imprints showed aggregates of large cells (blasts) with evidence of stromal changes and myelofibrosis on trephine biopsy. Following IHC on trephine biopsy, 18 out of 22 cases which had aparticulate aspirate were further classified into acute myeloid leukemia (AML) (5 cases), B-ALL (11 cases), and T-ALL (2 cases). The morphological and immunohistochemical features of AML are depicted in Figure 1. Among the seven cases which were scant particulate, IHC further categorized into four cases of AML, 2 B-ALL, and one T-ALL. Thus, IHC could accurately subtype $86.2 \%$ of the cases $(95 \%$ confidence interval $68.34 \%-96.11 \%$ ). Four cases which could not be subtyped on IHC were positive only for CD34 and negative for both lymphoid and myeloid markers. The subtyping of acute leukemia after cytochemistry and IHC is shown in Table 2. All the cases of ALL were positive for TdT and $66.67 \%$ of the cases were positive for CD34. Majority of the ALL were of B-cell lineage $(81.25 \%)$ and almost half of the B-ALL were common acute lymphoblastic leukemia-associated antigen positive. Figures 2 and 3 show morphological and immunohistochemical features of $\mathrm{B}$ and T-ALL. One case was negative for SBB on cytochemistry but showed positivity for MPO on trephine biopsy. TdT was also expressed in some of the cells. B- and T-cell markers were negative. Further IHC done with CD117 was also positive. Hence, it was categorized as AML with minimal 

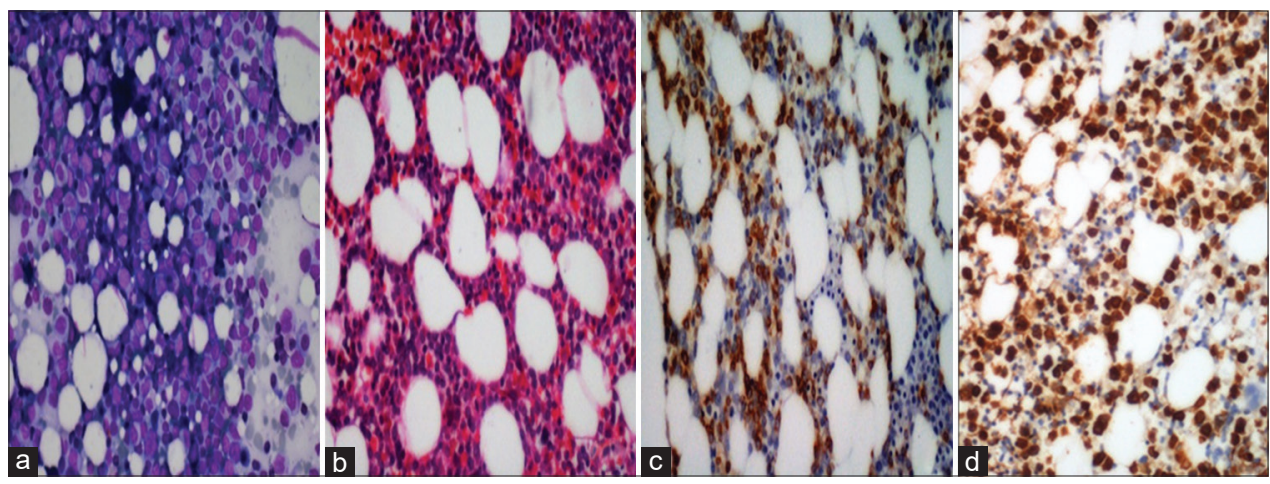

Figure 1: Acute myeloid leukemia: (a) Trephine imprints show prominence of blasts (Giemsa, $\times 100$ ); (b) Interstitial prominence of blasts on trephine biopsy ( $\mathrm{H}$ and E, $\times 100$ ); (c and d) CD34 and MPO is positive in the blasts (poly horse radish peroxidase, $\times 100$ [c] and $\times 200$ [d])

\begin{tabular}{|c|c|c|c|c|}
\hline Bone marrow aspirate & Diagnosis on BMA/imprint & $\begin{array}{c}\text { Number of } \\
\text { cases }\end{array}$ & $\begin{array}{l}\text { Definite diagnosis after } \\
\text { cytochemistry }\end{array}$ & $\begin{array}{l}\text { Definite diagnosis after IHC on } \\
\text { trephine biopsy }\end{array}$ \\
\hline \multirow[t]{12}{*}{ Aparticulate $(n=22)$} & Acute leukemia & 14 & ALL - 4 & AML - 3 \\
\hline & & & Noncontributory - 5 & B-ALL - 5 \\
\hline & & & & CALLA positive B ALL - 3 \\
\hline & & & & T-ALL - 1 \\
\hline & & & & Acute leukemia unclassifiable - 2 \\
\hline & Suspicious of leukemia & 6 & Noncontributory - 2 & AML - 2 \\
\hline & & & & CALLA positive B ALL - 1 \\
\hline & & & & Acute leukemia unclassifiable - 1 \\
\hline & & & & B-ALL - 1 \\
\hline & & & & T-ALL - 1 \\
\hline & Hypocellular marrow & 2 & & CALLA positive B ALL - 1 \\
\hline & & & & $\begin{array}{l}\text { Acute leukemia with myelofibrosis } \\
-1\end{array}$ \\
\hline \multirow[t]{6}{*}{ Scant particulate $(n=7)$} & Acute leukemia & 2 & Noncontributory - 6 & AML - 1 \\
\hline & & & & B-ALL - 1 \\
\hline & Suspicious of leukemia & 3 & & AML - 2 \\
\hline & & & & T-ALL - 1 \\
\hline & Hypocellular marrow & 2 & & CALLA positive B-ALL - 1 \\
\hline & & & & AML - 1 \\
\hline
\end{tabular}

BMA - Bone marrow aspirate; ALL - Acute lymphoblastic leukemia; AML - Acute myeloblastic leukemias; CALLA - Common acute lymphoblastic leukemia-associated antigen

differentiation. The results of IHC in various subtypes of acute leukemia are summarized in Table 3.

\section{Discussion}

In this era of personalized medicine, it is imperative to subtype acute leukemia for significant therapeutic implications and prognostic stratification. Immunophenotyping of leukemic blasts are essential for the confirmation of diagnosis and assessing specific lineage. Flow cytometry is the method of choice because of short turnaround time, ability to quantify the percentage of blasts, and detect multiple antigens using different flurochrome labeled antibodies. ${ }^{[3,4]}$ On the other hand, IHC was not preferred in the past due to inconsistent results. ${ }^{[5]}$ The improved antigen retrieval and staining techniques have changed the concept, and now IHC on trephine biopsy is recognized as an universally accessible method. ${ }^{[6-8]}$ It can be done on archival tissues and is most suitable when a dry tap is yielded on aspiration. ${ }^{[9]}$ Although it gives a semi-quantitative results, morphological correlation and ability to assess nonviable cells are an added advantage. It obviates the need of a repeat procedure to obtain the fresh samples for flow cytometry in all unsuspected cases where extra samples for ancillary studies are not routinely collected.

IHC is helpful when the morphology of the blasts is undifferentiated and cytochemical stains are noncontributory. In ALL, CD34 and TdT are expressed in $95 \%$ and $85 \%-90 \%$, respectively. ${ }^{[9]} \mathrm{TdT}$ is a marker for 


\begin{tabular}{lccccccccc}
\hline \multicolumn{7}{c}{ Table 3: Immunohistochemistry results in different subtypes of acute leukemia } \\
\hline Diagnosis & Number of cases & CD34 & MPO & CD117 & CD3 & CD20 & PAX5 & Tdt & CD10 \\
\hline AML & 9 & $9 / 9$ & $9 / 9$ & $3 / 7$ & $0 / 9$ & $0 / 9$ & $0 / 9$ & $1 / 9$ & $0 / 9$ \\
B-ALL & 13 & $6 / 9$ & $0 / 13$ & $0 / 2$ & $0 / 13$ & $8 / 11$ & $5 / 5$ & $13 / 13$ & $6 / 13$ \\
T-ALL & 3 & $2 / 3$ & $0 / 3$ & $0 / 1$ & $3 / 3$ & $0 / 3$ & $0 / 3$ & $3 / 3$ & $0 / 3$ \\
Acute leukemia - unclassified & 4 & $4 / 4$ & $0 / 4$ & - & $0 / 4$ & $0 / 4$ & $0 / 4$ & $0 / 4$ & $0 / 4$ \\
\hline
\end{tabular}

ALL - Acute lymphoblastic leukemia; AML - Acute myeloblastic leukemias

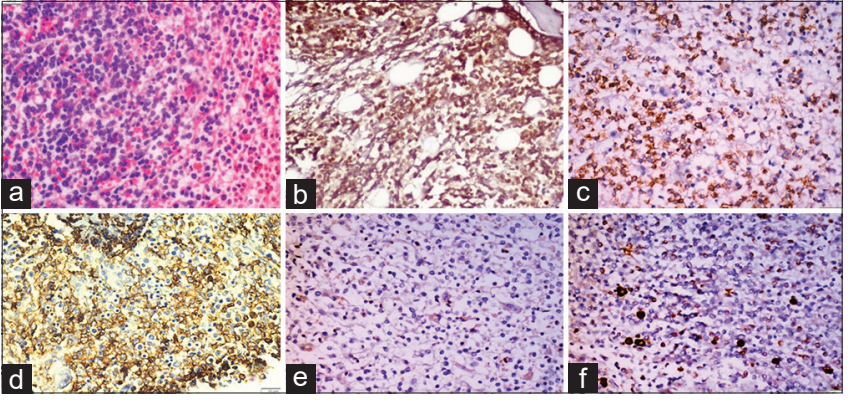

Figure 2: B-acute lymphoblastic leukemia: (a) Trephine biopsy show sheets of monomorphic round cells $(H$ and $E, \times 100)$. (b-d) Cells are positive for TdT, CD20, and CD10 (poly horse radish peroxidase, $\times 100$ ). (e and f) Cells are negative for CD3 and MPO (poly horse radish peroxidase, $\times 100$ )

immature lymphoid cells. Orazi et al. accounted for 97\% positivity in pre-B and T-ALL. ${ }^{[10]}$ The European Group for the Immunological Characterization of Leukemias (EGIL), US-Canadian Consensus Group (USCC), and British Committee for Standards in Hematology (BCSH) recommends CD19, CD20, and CD10 for the detection of lymphoblasts of B-cell type. CD19 is not preferred for IHC and CD10 can be detected both by flow cytometry and IHC. ${ }^{[9]}$ CD20 a marker for B-cell lineage is often found to be negative or weakly positive. In such cases, other B-cell markers such as PAX5 or CD79a can be used. ${ }^{[1,12]}$ In the present study, we have used PAX5 in cases where CD20 was negative or weakly positive. Literature reveals that antiCD3 is expressed well by T-lymphocytes on paraffin sections. ${ }^{[13]}$ The other T-cell antigens (CD2, CD5, and CD7) are not lineage specific and can be aberrantly expressed in AML. Hence a diagnosis of T-ALL requires the expression of TdT and CD3. Hence, a comprehensive panel of CD3, CD20, PAX5, and TdT was used in the present study. The results of the four cases of ALL diagnosed on morphology and cytochemistry corroborated well with the IHC findings.

The EGIL, USCC, and BCSH advocate the use of antiMPO, CD13, and CD33 to detect myeloid blasts. ${ }^{[14]}$ CD13 and CD33 are detected by flow cytometry and are not available as IHC in most of the laboratories. AntiMPO detected by IHC has a high specificity for myeloblasts. ${ }^{[13]}$

The sensitivity of IHC in subtyping acute leukemia in the present study $(86.2 \%)$ is comparable with Subashchandrabose et al. (90\%) and Arber et al. $(96 \%) \cdot{ }^{[5,15]}$ Four cases which could not be subtyped on IHC in the present study might be either AML with minimal differentiation or monocytic lineage. Expression of CD117

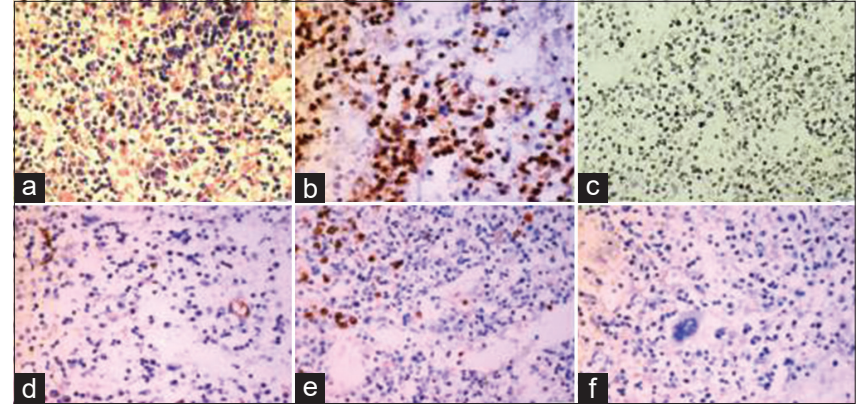

Figure 3: T-acute lymphoblastic leukemia: (a) Marrow is replaced by monomorphous cells with round vesicular nucleus and scant cytoplasm (H and E $\times 200$ ); (b) and $\times 100$ (c) Cells are positive for TdT and CD3 (poly horse radish peroxidase, $\times 200$; (d-f) Cells are negative for CD34, MPO and CD20 (poly horse radish peroxidase, $\times 100$ )

and TdT in the absence of CD79a, PAX-5, CD3, and MPO favors a diagnosis of AML with minimal differentiation. AML with myeloblastic component expresses MPO. Monoblasts are MPO negative and express CD68. Al Gwaiz and Bassioni in their study compared the results of flow cytometry and IHC and found high concordance for CD3 and CD10 but not CD20 in ALL. ${ }^{[2]}$

\section{Conclusion}

IHC on trephine biopsy facilitates the confirmation and subtyping of leukemia in more than $85 \%$ cases. A limited panel of antibodies using CD34, MPO, TdT, CD3 CD20, PAX 5, and CD10 can be used for sub-typing of leukemias in trephine biopsy sections, especially in centers with limited resources. It is useful alternative to flow cytometry, especially in cases where aspirate/peripheral blood is not available and prevents a repeat procedure to obtain material for immunophenotyping.

Financial support and sponsorship

Nil.

\section{Conflicts of interest}

There are no conflicts of interest.

\section{References}

1. Arber DA, Brunning RD, Le Beau MM, Falini B, Vardiman JW, Porwit A, et al. Acute myeloid leukemia with recurrent genetic abnormalities. In: Swerdlow SH, Campo E, Harris NL, Jafee ES, Pileri SA, Stein H, editors. WHO Classification of Tumors of Haematopoietic and Lymphoid Tissues. Revised $4^{\text {th }}$ ed.. France: International Agency for Research on Cancer; 2017. p. 129-30.

2. Al Gwaiz LA, Bassioni W. Immunophenotyping of acute 
lymphoblastic leukemia using immunohistochemistry in bone marrow biopsy specimens. Histol Histopathol 2008;23:1223-8.

3. Kroft SH. Role of flow cytometry in pediatric hematopathology. Am J Clin Pathol 2004;122 Suppl:S19-32.

4. Dunphy $\mathrm{CH}$. Gene expression profiling data in lymphoma and leukemia: Review of the literature and extrapolation of pertinent clinical applications. Arch Pathol Lab Med 2006;130:483-520.

5. Arber DA, Jenkins KA. Paraffin section immunophenotyping of acute leukemias in bone marrow specimens. Am J Clin Pathol 1996;106:462-8.

6. Herman GE, Elfont EA, Floyd AD. Overview of automated immunostainers. Methods Mol Biol 1994;34:383-403.

7. Cuevas EC, Bateman AC, Wilkins BS, Johnson PA, Williams $\mathrm{JH}$, Lee $\mathrm{AH}$, et al. Microwave antigen retrieval in immunocytochemistry: A study of 80 antibodies. J Clin Pathol 1994;47:448-52.

8. Blumenthal D, Gluck M, Louis KS, Stoto MA, Wise D. University-industry research relationships in biotechnology: Implications for the university. Science 1986;232:1361-6.

9. Olsen RJ, Chang CC, Herrick JL, Zu Y, Ehsan A. Acute leukemia immunohistochemistry: A systematic diagnostic approach. Arch Pathol Lab Med 2008;132:462-75.
10. Orazi A, Cotton J, Cattoretti G, Kotylo PK, John K, Manning JT, et al. Terminal deoxynucleotidyl transferase staining in acute leukemia and normal bone marrow in routinely processed paraffin sections. Am J Clin Pathol 1994;102:640-5.

11. Chu PG, Loera S, Huang Q, Weiss LM. Lineage determination of CD20- B-Cell neoplasms: An immunohistochemical study. Am J Clin Pathol 2006;126:534-44.

12. Gibson SE, Dong HY, Advani AS, Hsi ED. Expression of the B cell-associated transcription factors PAX5, OCT-2, and BOB.1 in acute myeloid leukemia: Associations with B-cell antigen expression and myelomonocytic maturation. Am J Clin Pathol 2006;126:916-24.

13. Chuang SS, Li CY. Useful panel of antibodies for the classification of acute leukemia by immunohistochemical methods in bone marrow trephine biopsy specimens. Am J Clin Pathol 1997;107:410-8.

14. Bain BJ. Leukemia Diagnosis. $4^{\text {th }}$ ed.. Singapore: Wiley-Blackwell; 2010. p. 64-113.

15. Subashchandrabose P, Ramiah Madanagopaal L, Subba Rao TM. Diagnosis and classification of acute leukemia in bone marrow trephine biopsies, utility of a selected panel of minimal immunohistochemical markers. Int $\mathrm{J}$ Hematol Oncol Stem Cell Res 2016;10:138-46. 